\title{
Temporal parameters as cues to phrasal boundaries: A comparison of processing by left- and right-hemisphere brain-damaged individuals ${ }^{\text {is }}$
}

\author{
Wendi A. Aasland and Shari R. Baum* \\ School of Communication Sciences and Disorders, McGill University, 1266 Pine Avenue West, Montréal, Que., Canada H3G 1 A8
}

Accepted 18 April 2003

\begin{abstract}
Two experiments were conducted to examine the ability of left- (LHD) and right-hemisphere-damaged (RHD) patients and normal controls to use temporal cues in rendering phrase grouping decisions. The phrase "pink and black and green" was manipulated to signal a boundary after "pink" or after "black" by altering pre-boundary word durations and pause durations at the boundary in a stepwise fashion. Stimuli were presented to listeners auditorily along with a card with three alternative groupings of colored squares from which to select the presented alternative. Results revealed that normal controls were able to use both temporal cues to identify the intended grouping. In contrast, LHD patients required longer than normal pause durations to consistently identify the intended grouping, suggesting a higher than normal threshold for perception of temporal prosodic cues. Surprisingly, the RHD patients exhibited great difficulty with the task, perhaps due to the limited acoustic cues available in the stimuli.
\end{abstract} (C) 2003 Elsevier Science (USA). All rights reserved.

Keywords: Prosody; Temporal processing; Speech perception; Brain damage; Cerebral dominance

\section{Introduction}

Speech prosody conveys both linguistic and affective information to listeners, mainly through variations in duration, fundamental frequency, and amplitude of the acoustic signal. A large number of investigations has demonstrated that individuals who have suffered either left (LHD) or right hemisphere damage (RHD) may exhibit deficits in the perception or interpretation of prosodic cues (see Baum \& Pell, 1999 for a review). With specific regard to linguistic prosody, a majority of studies has shown receptive impairments following LHD (Baum, Kelsch Daniloff, Daniloff, \& Lewis, 1982; Em-

\footnotetext{
Wendi A. Aasland, School of Communication Sciences and Disorders, McGill University; Shari R. Baum, School of Communication Sciences and Disorders, McGill University.This research was supported by an operating grant from the Canadian Institutes of Health Research as well as by a CIHR studentship. Portions of this work were presented at the September 2002 meeting of the International Conference on Spoken Language Processing, Denver, Colorado, and published in the Proceedings of the ISCLP Conference.

*Corresponding author.
}

morey, 1987; Gandour et al., 1992; Pell \& Baum, 1997) and fewer deficits subsequent to RHD (Behrens, 1988; Emmorey, 1987; Gandour, Dechongkit, Ponglorpisit, Khunadorn, \& Boongird, 1993, 1995; but cf. Blumstein \& Cooper, 1974; Brådvik et al., 1991). Many of these (and other) studies have noted particular impairments in LHD individuals in the perception of temporal parameters, as signals of lexical or emphatic stress or sentence modality (e.g., Tallal \& Newcombe, 1978; Van Lancker $\&$ Sidtis, 1992). Interestingly, in parallel with findings for speech timing, impairments subsequent to LHD have been found for non-speech signals as well (e.g., Carmon \& Nachshon, 1971; Robin, Tranel, \& Damasio, 1990). In addition, functional neuroimaging studies and studies using intracerebral neural recordings support a left hemisphere specialization or superiority for temporal processing versus a right hemisphere superiority for spectral processing (e.g., Liégeois-Chauvel, de Graaf, \& Laguitton, 1999, 2001; Zatorre, 1988; Zatorre, Evans, Meyer, \& Gjedde, 1992; see also Efron, 1963; but cf. Langner, Sams, Heil, \& Schulze, 1997). Recent data have even shown anatomical differences between the auditory 
cortices of the two hemispheres that may support the different processing attributes of the left and right hemispheres (Penhune, Zatorre, \& MacDonald, 1996). Thus, a great deal of evidence exists for the preferential processing of temporal parameters in the left hemisphere, and relatedly, impairments in temporal processing subsequent to LHD.

Nonetheless, the situation with respect to speech prosody has proven to be somewhat more complex, as the linguistic functional load of a stimulus seems to interact with its acoustic characteristics (and perhaps other parameters such as domain of processing (e.g., Behrens, 1989; Gandour et al., 1993, 1989)) in determining which hemisphere is most implicated in prosodic perception (see Baum \& Pell, 1999 for review). Moreover, even when deficits in processing temporal aspects of prosody appear consistent, it remains unclear whether the impairments are due to an inability to perceive timing cues, difficulty mapping the acoustic parameters to linguistically significant contrasts, or a reduced sensitivity to the temporal distinctions resulting in higher thresholds of perception (see e.g., Johnsrude, Penhune, \& Zatorre, 2000; Robin et al., 1990). The present study addresses the last of these possibilities within a specific domain of linguistic prosodic cues.

Whereas numerous studies have explored stress contrasts and sentence modality (i.e., declarative versus interrogative or imperative sentence types) as cued by prosodic parameters, only a relatively small number of investigations has focused on the perception of prosody as a cue to syntactic disambiguation in brain-damaged individuals (Baum, Pell, Leonard, \& Gordon, 1997; Grosjean \& Hirt, 1996; Perkins, Baran, \& Gandour, 1996). Most directly relevant to the present study, in an initial investigation, Baum et al. (1997) examined the ability of LHD and RHD patients to identify phrase boundaries (specifically phrasal groupings) through prosodic cues. ${ }^{1}$ Using three arrangements of colored squares ("pink and black and green"-_PBG; "[pink and black] and green"-PB_G; and "pink and [black and green]"-P_BG), groups of LHD and RHD patients as well as normal controls were required to identify the appropriate grouping associated with a presented stimulus. Results revealed that both LHD and RHD patients were comparably impaired relative to the normal controls in the identification of the phrase groupings, although individual variability was notable within both groups, with three subjects in each group performing at ceiling (Baum et al., 1997). While the perceptual findings for the RHD patients were somewhat surprising, they are not without precedent (e.g., Blumstein \& Cooper, 1974; Brådvik et al., 1991; Bryan, 1989; Weintraub,

\footnotetext{
${ }^{1}$ A production experiment was included as well, but will not be described here.
}

Mesulam, \& Kramer, 1981). Further, as suggested by Van Lancker and Sidtis (1992), impairments in prosodic perception in LHD and RHD patients may be due to different perceptual anomalies (i.e., reliance on different prosodic cues; but cf. Baum, 1998; Pell \& Baum, 1997).

The experiments presented here investigate the relationship between two temporal prosodic cues in identifying phrasal boundaries-the duration of the preboundary word and of the pause at the phrase boundary-both of which have been shown to be important in disambiguating phrasal groupings (Price, Ostendorf, Shattuck-Hufnagel, \& Fong, 1991; Scott, 1982; Streeter, 1978). These two cues were varied in combination to determine the critical durations of each necessary for LHD and RHD patients, relative to normal controls (NC), to identify phrasal boundaries. To this end, an initial set of stimuli was generated in which pause and keyword duration were systematically manipulated using edited natural speech, and combined to create what could be considered the equivalent of 10 pause continua and 10 keyword continua (five each ranging from more PBG-like to more $\mathrm{P} \_$BG-like and five each ranging from more PBG-like to more PB_Glike). Based on our previous findings and the well-established impairments in LHD patients in temporal processing (e.g., Robin et al., 1990; Van Lancker \& Sidtis, 1992), it was anticipated that the LHD patients would require longer than normal pause and keyword durations to identify the phrase groupings. Given the RHD patients' performance in our initial study, they may also exhibit impairments compared to normal controls in identification of phrase boundaries; however, if RHD patients are better able to make use of temporal cues to prosody (e.g., Van Lancker \& Sidtis, 1992), the critical durations required for boundary identification for these patients should be closer to normal.

\section{Experiment 1}

\subsection{Methods}

\subsubsection{Subjects}

Three groups of subjects participated in the current experiment: 10 LHD non-fluent aphasic patients, nine RHD patients, and 10 age-matched non-brain-damaged control subjects. All subjects were right-handed native speakers of English who passed an audiometric screening at $<35 \mathrm{~dB} \mathrm{HL}$ at the frequencies .5, 1 , and $2 \mathrm{kHz}$ in the better ear. Brain-damaged patients had all suffered a single, unilateral cerebrovascular accident (CVA; confirmed by $\mathrm{CT}$ or MRI) and underwent a series of screening tests that varied depending on their lesion lateralization. Background information on the participants appears in Table 1. 
Table 1

Background information on patients with brain damage

\begin{tabular}{|c|c|c|c|c|c|}
\hline $\begin{array}{l}\text { Experiment } 1 \\
\text { subjects }\end{array}$ & $\begin{array}{l}\text { Experiment } 2 \\
\text { subjects }\end{array}$ & Age & $\mathrm{MPO}^{\mathrm{a}}$ & Lesion site & Diagnostic characteristics \\
\hline \multicolumn{6}{|c|}{ Left hemisphere damaged patients } \\
\hline LHD 1 & LHD 1 & 68 & 78 & Left fronto-temporo-parietal & Moderate-severe non-fluent aphasia \\
\hline LHD 2 & LHD 2 & 72 & 79 & Left parietal & Non-fluent aphasia \\
\hline LHD 3 & - & 80 & 33 & Left MCA territory & Transcortical motor aphasia \\
\hline LHD 4 & LHD 4 & 83 & 75 & N/A & Non-fluent aphasia (recovered) \\
\hline LHD 5 & LHD 5 & 82 & 13 & Left MCA & Non-fluent aphasia \\
\hline LHD 6 & - & 74 & 32 & Left MCA & Severe non-fluent mixed aphasia \\
\hline LHD 7 & LHD 7 & 80 & 56 & Left frontal & Mild non-fluent aphasia \\
\hline LHD 8 & - & 73 & 113 & Left temporo-parietal & Severe non-fluent aphasia \\
\hline LHD 9 & LHD 9 & 48 & 101 & Left fronto-parietal & Non-fluent aphasia \\
\hline LHD 10 & LHD 10 & 76 & 50 & Left MCA & Non-fluent aphasia \\
\hline- & LHD 11 & 53 & 160 & Left parietal & Mild-moderate non-fluent aphasia \\
\hline- & LHD 12 & 69 & 71 & Left fronto-parietal & Non-fluent aphasia, anomia, apraxia of speech \\
\hline- & LHD 13 & 57 & 18 & $\begin{array}{l}\text { Massive ischemia, left } \\
\text { carotid artery }\end{array}$ & Moderate-severe non-fluent aphasia \\
\hline \multicolumn{6}{|c|}{ Right hemisphere damaged patients } \\
\hline RHD 1 & RHD 1 & 43 & 41 & Right MCA & Minimal dysarthria noticed initially \\
\hline RHD 2 & RHD 2 & 90 & 39 & N/A & Impulsive, left neglect \\
\hline RHD 3 & RHD 3 & 34 & 58 & Right MCA & N/A \\
\hline RHD 4 & RHD 4 & 67 & 58 & N/A & $\begin{array}{l}\text { Impaired comprehension of inferences and figurative } \\
\text { language, mild impairment in processing emotional } \\
\text { prosody. Left neglect }\end{array}$ \\
\hline RHD 5 & RHD 5 & 59 & 121 & $\begin{array}{l}\text { Right posterior } \\
\text { communicating artery }\end{array}$ & $\begin{array}{l}\text { Flat affect, rapid speech, impulsivity, sudden topic } \\
\text { changes, inappropriate topics, mild left neglect }\end{array}$ \\
\hline RHD 6 & RHD 6 & 66 & 62 & $\begin{array}{l}\text { Right internal capsule, right } \\
\text { basal ganglia }\end{array}$ & Flat affect \\
\hline RHD 7 & - & 72 & 50 & Right parietal & Emotionally labile, mild dysarthria \\
\hline RHD 8 & - & 70 & 45 & Subcortical-right thalamus & Slowed speech \\
\hline RHD 9 & RHD 9 & 79 & 37 & Right temporo-parietal & $\begin{array}{l}\text { Dysphagia, mild dysarthria and mild anomia, } \\
\text { left neglect }\end{array}$ \\
\hline- & RHD 10 & 89 & 107 & Right MCA & Left neglect \\
\hline- & RHD 11 & 64 & 8 & N/A & N/A \\
\hline
\end{tabular}

${ }^{\mathrm{a}}$ Months post-onset.

\subsubsection{Stimuli}

Three versions of the phrase "pink and black and green" were recorded onto DAT by an adult male native speaker of English. Each version represented a different phrasal grouping, corresponding to the grouping of three colored squares (i.e., "pink and black and green"; "[pink and black] and green"; and "pink and [black and green]"). These recordings were digitized at a rate of $20 \mathrm{k}$ samples/s with a $9 \mathrm{kHz}$ low-pass filter and 12-bit quantization using the BLISS speech analysis system (Mertus, 1989). Measurements were made of each word and pause within each phrase as a baseline from which to generate the experimental stimuli. The original keyword "pink" ranged in duration from 233 to $443 \mathrm{~ms}$; the original "black" productions ranged from 284 to $417 \mathrm{~ms}$; and the original "green" varied very little, from 371 to $374 \mathrm{~ms}$. Pauses ranged from $0 \mathrm{~ms}$ to an extreme of $259 \mathrm{~ms}$. Individual keywords were selected for editing to create a series of stimuli with the following temporal characteristics. The keyword "pink" ranged from 286 to $443 \mathrm{~ms}$ in five approximately $40 \mathrm{~ms}$ steps (labeled P1 through P5); the keyword "black" was similarly edited to vary between 284 and $441 \mathrm{~ms}$ in five approximately $40 \mathrm{~ms}$ steps (labeled B1 through B5). Five pauses of 0 to $160 \mathrm{~ms}$ (in $40 \mathrm{~ms}$ steps, i.e., $0,40,80,120$, and $160 \mathrm{~ms}$ ) were also created. An original "green" (371 ms) and an original "and" (163 ms) were selected to be used with each continuum created. The keywords ("pink" and "black") were edited by removing or reiterating adjacent pitch periods from the midpoint (steady-state) of the vowel; cuts were made at zero crossings to avoid extraneous clicks. The resulting individual words and pauses were concatenated to create a total of 49 unique stimuli: each duration of "pink" $(n=5)$ paired with each pause length $(n=5)$, appended to the shortest "black" and zero pause; and each duration of "black" $(n=5)$ paired with each pause length $(n=5)$, appended to the shortest "pink" and zero pause. The original "and" and "green" were used throughout. (See Appendix A for an example of these continua for the keyword "pink.")

\subsubsection{Procedure}

A test tape was made which included five repetitions of each of the phrasal stimuli in random order. Simultaneous with presentation of the auditory stimulus over 
closed headphones at a comfortable listening level, a card depicting the three possible arrangements of colored squares was presented to the subject. The three alternatives were organized vertically and every possible order of options was randomly paired with an auditory stimulus. Subjects indicated their responses by pointing to the appropriate grouping represented by the stimulus; responses were recorded by the experimenter for later analysis.

\subsection{Results and discussion}

The number of $\mathrm{P} \_\mathrm{BG}$ responses produced by each individual listener for each stimulus was computed for the continua which were expected to yield an increasing number of P_BG responses, i.e., those for which the keyword "pink" and the following pause were manipulated. The data (converted to percentages) for these "P_BG" continua are illustrated in Figs. 1 and 2. In a similar fashion, the number of PB_G responses made by each subject for each stimulus was computed for the continua which were expected to yield an increasing

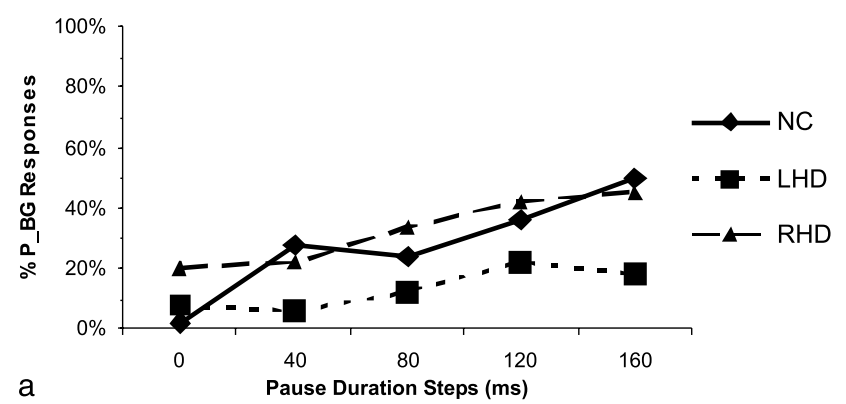

a

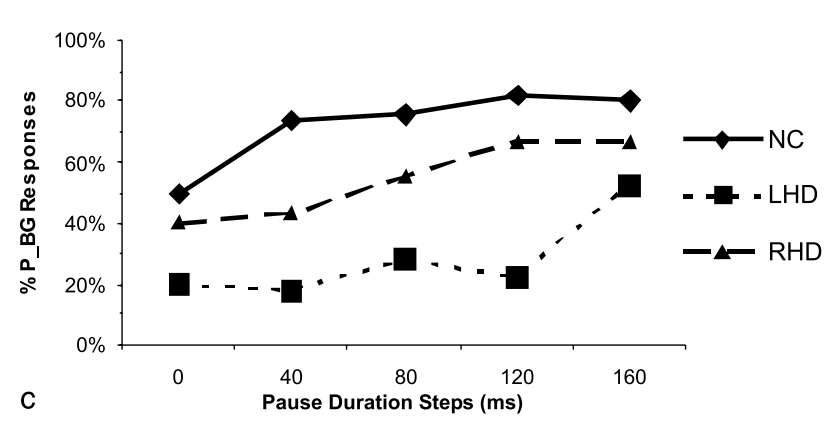

number of PB_G responses, i.e., those for which the keyword "black" and the following pause were varied. These data are illustrated in Figs. 3 and 4.

As may be seen in the results for the $\mathrm{P}_{-} \mathrm{BG}$ set of continua, with the shortest keyword "pink" (P1) continuum, not even the longest pause $(160 \mathrm{~ms})$ led to a large number of $\mathrm{P} \_\mathrm{BG}$ responses for any group (Fig. 1a). With longer keyword "pink" durations, the increases in pause length yielded more consistent P_BG responses, with $>70 \%$ P_BG responses for all stimuli with "pink" keyword durations of at least $364 \mathrm{~ms}$ (P3) and a minimum pause of $40 \mathrm{~ms}$ for the normal control subjects. For the longest keyword duration (Fig. 1e), even with a $0 \mathrm{~ms}$ pause, normal controls selected the P_BG grouping approximately $70 \%$ of the time. In contrast, neither patient group showed a marked consistency in P_BG responses across the pause continua (Fig. 1a-e), with the exception that the RHD group achieved approximately $70 \%$ consistency for the longest two pause durations ( $71 \%$ at $120 \mathrm{~ms}$ and $69 \%$ at $160 \mathrm{~ms}$ ) presented with the longest "pink" duration (Fig. 1e). It appears that the RHD group benefited from the
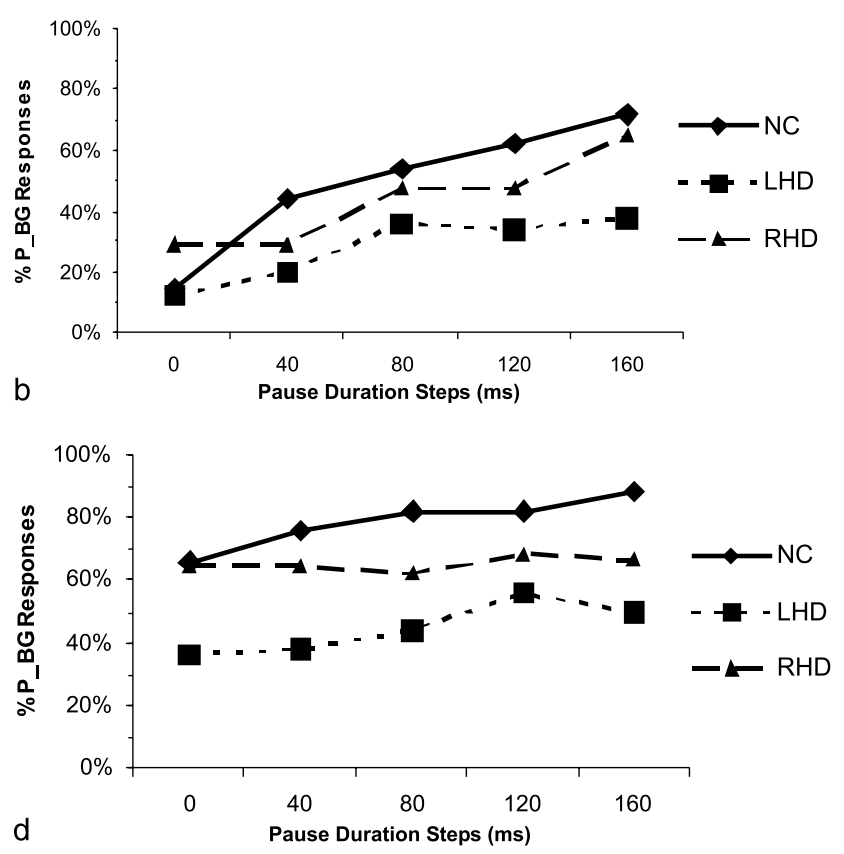

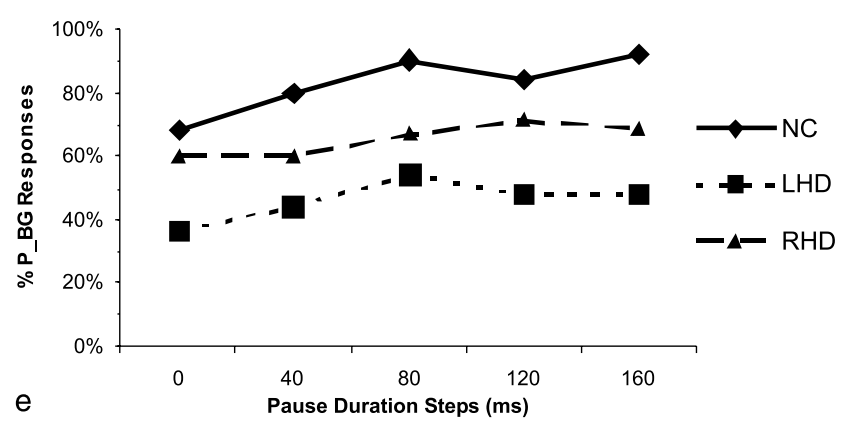

Fig. 1. Percent P_BG responses by normal control (NC), RHD, and LHD subjects across the pause duration continuum (0-160 ms post-keyword pause), for each duration of the keyword "pink" ( $\mathrm{a}=\mathrm{P} 1, \mathrm{~b}=\mathrm{P} 2, \mathrm{c}=\mathrm{P} 3, \mathrm{~d}=\mathrm{P} 4, \mathrm{e}=\mathrm{P} 5)$. 

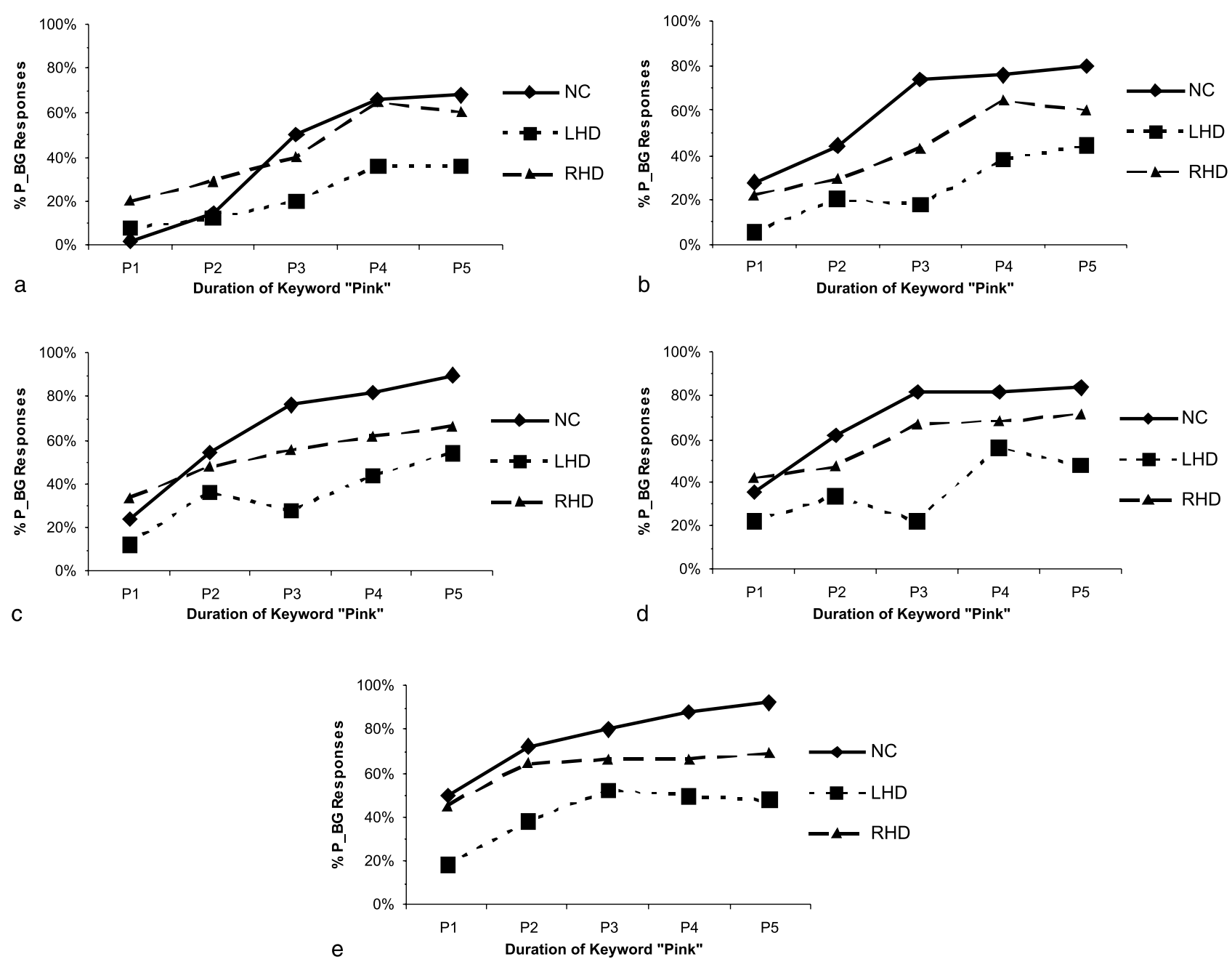

Fig. 2. Percent P_BG responses by NC, RHD, and LHD subjects across the keyword duration continuum for the keyword "pink" (P1 $=286 \mathrm{ms,}$ $\mathrm{P} 2=325 \mathrm{~ms}, \mathrm{P} 3=364 \mathrm{~ms}, \mathrm{P} 4=404 \mathrm{~ms}$, and $\mathrm{P} 5=443 \mathrm{~ms})$, for each pause duration $(\mathrm{a}=0 \mathrm{~ms}, \mathrm{~b}=40 \mathrm{~ms}, \mathrm{c}=80 \mathrm{~ms}, \mathrm{~d}=120 \mathrm{~ms}, \mathrm{e}=160 \mathrm{~ms}$ pause).

combination of temporal cues, but only when these cues approached values found in normal speech. With pause held constant and keyword duration increasing, normal controls achieved $\geqslant 70 \%$ P_BG responses with a pause of $40 \mathrm{~ms}$ and keyword of $364 \mathrm{~ms}$ (P3). With a $160 \mathrm{~ms}$ pause, the duration of "pink" only needed to be $325 \mathrm{~ms}$ (P2) to reach $\geqslant 70 \%$ P_BG responses. Again, neither patient group consistently $(\geqslant 70 \%)$ responded $\mathrm{P} \_\mathrm{BG}$ for any stimulus, with the exception of the RHD group's borderline responses for the two longest pauses and the longest keyword duration.

The raw data (number of $\mathrm{P}_{-} \mathrm{BG}$ responses) were submitted to a series of Group $\times$ Step analyses of variance (ANOVAs) - one for each continuum. Of particular interest were any Group $\times$ Step interactions that might emerge. A list of the main effects appears in Appendix B. Only two of the ANOVAs yielded Group$\times$ Step interactions: The pause continuum created with the $364 \mathrm{~ms}$ (P3) "pink" keyword $(F(8,104)=2.085$, $p<.05)$ and the "pink" continuum created with the
$120 \mathrm{~ms}$ pause $(F(8,104)=2.580, p<.02)$. Post hoc analysis of the first interaction using the Newman-Keuls procedure $(p<.05)$ revealed significant differences between the number of $\mathrm{P} \_\mathrm{BG}$ responses by the normal controls compared to the LHD patients at each continuum step. A significant difference also emerged between the normal controls and the RHD subjects for step 2 only. No differences between the patient groups reached significance. For the second Group $\times$ Step interaction, post hoc analysis yielded significant differences between the normal controls and the LHD patients at all but the first continuum step, and no differences between the normal controls and the RHD patient group. The LHD and RHD groups differed only for responses to step 3 of the continuum, with RHD patients producing more $\mathrm{P}_{-} \mathrm{BG}$ responses (i.e., more like the normal controls).

With regard to the "PB_G continua," it is evident in the graphs (Figs. 3 and 4) that, for the shortest two durations of the keyword "black" (B1, B2), not even the 

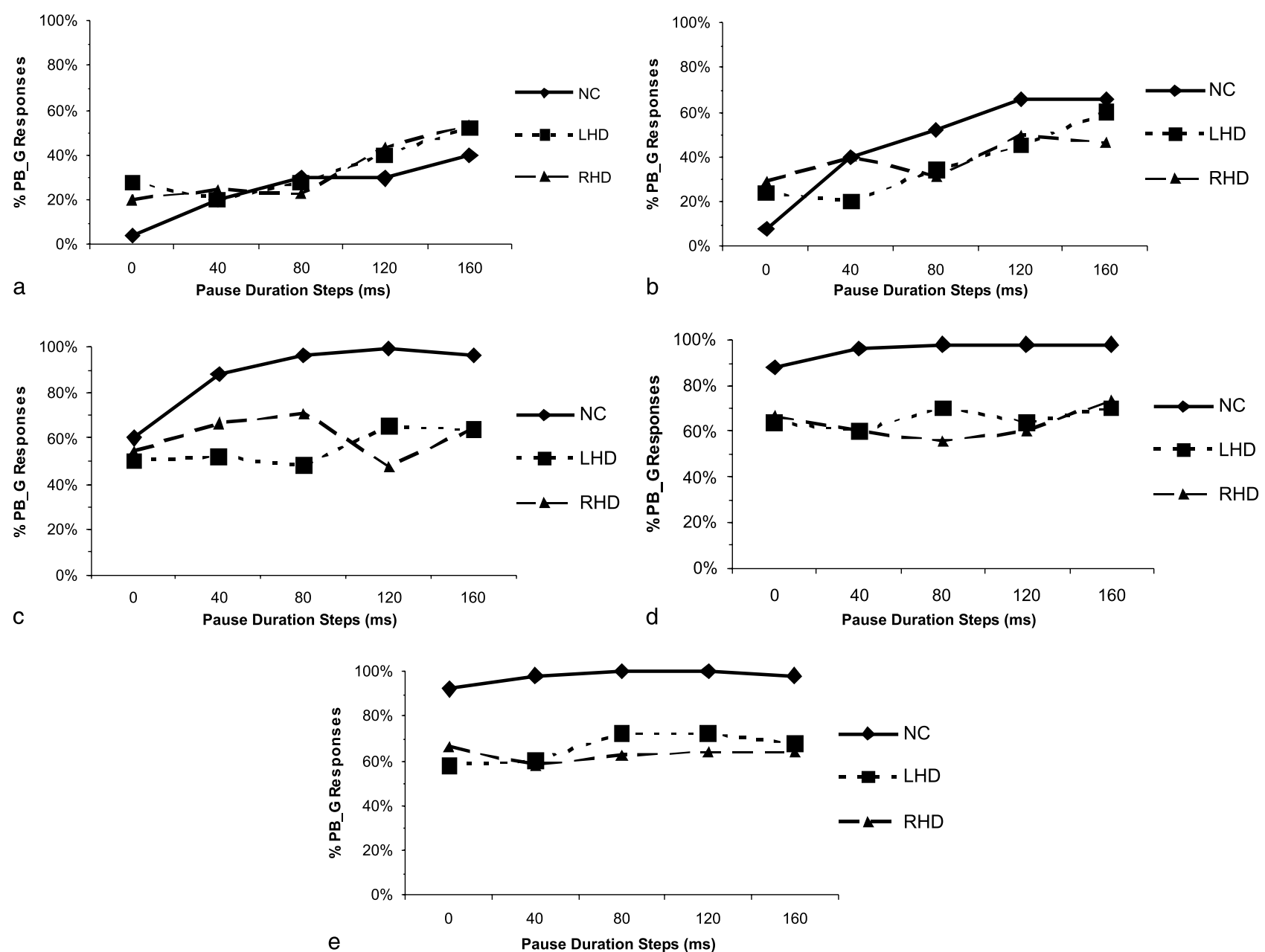

Fig. 3. Percent PB_G responses by normal control (NC), RHD, and LHD subjects across the pause duration continuum (0-160 ms post-keyword pause), for each duration of the keyword "black" $(\mathrm{a}=\mathrm{B} 1, \mathrm{~b}=\mathrm{B} 2, \mathrm{c}=\mathrm{B} 3, \mathrm{~d}=\mathrm{B} 4, \mathrm{e}=\mathrm{B} 5)$.

longest pause yielded consistent PB_G responses for any group. For longer "black" durations, very short pauses of 0 or $40 \mathrm{~ms}$ yielded $>70 \%$ PB_G responses for the normal controls, with performance near $100 \%$ PB_G responses with slightly longer pauses or even with a $0 \mathrm{~ms}$ pause at the longest keyword duration. Unlike the P_BG results, the LHD patient group reached $\geqslant 70 \%$ PB_G responses for the longest two keywords with pauses of at least $80 \mathrm{~ms}$, although these durations are longer than those required by the normal listeners. The RHD group only achieved $>70 \%$ PB_G responses for the $80 \mathrm{~ms}$ pause $/ 363 \mathrm{~ms}$ (B3) keyword and the $160 \mathrm{~ms}$ pause/402 ms (B4) keyword steps. This pattern clearly does not reflect consistent improvement with increasing keyword or pause durations; rather the relatively good performance on selected stimuli appears somewhat idiosyncratic.

With pause held constant and keyword duration increasing (Fig. 4), normal controls reached $>70 \%$ PB_G responses with a pause of $0 \mathrm{~ms}$ and keyword of $402 \mathrm{~ms}$ (B4). With longer pauses, keyword durations of $363 \mathrm{~ms}$
(B3) yielded performance near 100\% PB_G responses for the NC subjects. For the LHD listeners, with pauses of $\geqslant 80 \mathrm{~ms}, \geqslant 70 \%$ PB_G responses were generally produced with the longest two keyword durations (B4 and B5) (although some variability was evident). Again, the RHD group achieved $>70 \%$ PB_G responses only in the two conditions mentioned above $(80 \mathrm{~ms}$ pause/ 363 ms (B3) "black"; 160 ms pause/402 ms (B4) "black" continuum).

The raw data (number of PB_G responses) were analyzed in a series of Group $\times$ Step ANOVAs. Main effects are again listed in Appendix B. As noted earlier, any Group $\times$ Step interactions that would emerge were of particular interest in the present investigation. Such interactions emerged in the pause continua created with the $324 \mathrm{~ms}(\mathrm{~B} 2)(F(8,104)=2.056, p<.05)$ and $363 \mathrm{~ms}$ (B3) $(F(8,104)=4.505, p<.001)$ keywords (Fig. 3bc). Post hoc analysis of the first interaction using the Newman-Keuls procedure revealed no significant group differences at any step. For the pause continuum created with the $363 \mathrm{~ms}$ (B3) keyword, however, significant 

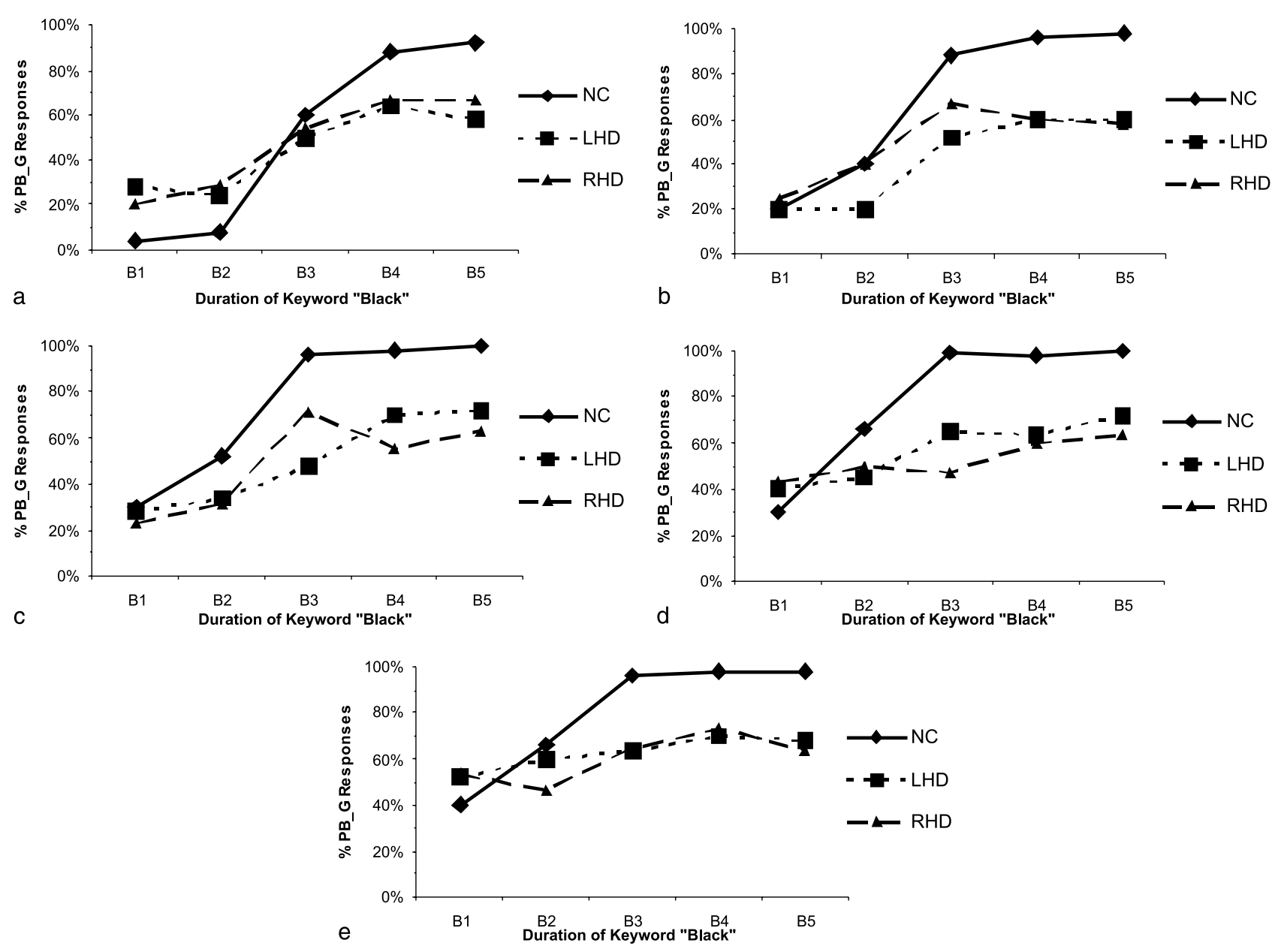

Fig. 4. Percent PB_G responses by NC, RHD, and LHD subjects across the keyword duration continuum for the keyword "black" (B1 $=284 \mathrm{~ms}$, $\mathrm{B} 2=324 \mathrm{~ms}, \mathrm{~B} 3=363 \mathrm{~ms}, \mathrm{~B} 4=402 \mathrm{~ms}, \mathrm{~B} 5=441 \mathrm{~ms})$, for each pause duration $(\mathrm{a}=0 \mathrm{~ms}, \mathrm{~b}=40 \mathrm{~ms}, \mathrm{c}=80 \mathrm{~ms}, \mathrm{~d}=120 \mathrm{~ms}, \mathrm{e}=160 \mathrm{~ms}$ pause).

differences were found between the normal controls and each patient group at all but the first continuum step; no differences emerged between the LHD and RHD groups. Group $\times$ Step interactions also emerged for all but one (with pause $=80 \mathrm{~ms}$ ) of the "black" continua, as illustrated in Fig. 4 (pause 0: $(F(8,104)=3.621, p<$ .001 ; pause $40: F(8,104)=2.492, p<.02$; and pause 120: $\quad F(8,104)=5.857, p<.001 ;$ pause $160: \quad(F(8$, $104)=2.388, p<.05)$. For the majority of continua, differences (based on post hoc analyses) emerged between normal controls and both patient groups for longer duration keywords only (i.e., continua steps 4 and 5 or 3, 4, and 5 only); the responses of the LHD and RHD patients did not differ significantly.

Given that there is normally heterogeneity of performance within patient groups (e.g., Baum et al., 1997), it is important to examine the performance of the individual subjects within each group. Among the LHD subjects, five (LHD 2, 5, 6, 7, and 8) performed similarly to normal subjects, with $>70 \%$ of responses in the intended direction with a pause of $40 \mathrm{~ms}$ and keyword duration of $363 \mathrm{~ms}$ (B3) for the PB_G continua. These subjects experienced somewhat more difficulty with the P_BG continua (as did the normal controls), with fewer subjects (LHD 6, 7, and 8) performing accurately ( $>70 \%$ of intended responses), and requiring more salient minimum cues (a pause of $160 \mathrm{~ms}$ and keyword duration of $364 \mathrm{~ms}$ (P3)) to achieve relatively consistent responding. In contrast, four other subjects (LHD 1, 3, 4, and 10) failed to show any marked improvement in performance, regardless of cue salience for both $\mathrm{P} \_\mathrm{BG}$ and PB_G continua. The remaining subject in this group (LHD 9) did show some increase in accuracy with the longer keyword and pause durations in both continua, at times achieving $>70 \%$, but this level of performance was sporadic. With regard to the RHD group, four of the nine subjects (RHD 2, 3, 5, and 8) usually achieved $>70 \%$ accuracy in their responses across all pause durations with a minimum keyword duration of $363 \mathrm{~ms}$ (B3) for the PB_G continua. Despite the relatively strong performance of these subjects, however, inexplicable drops in accuracy often occurred; for example, one subject displayed $100 \%$ expected performance at one step, but dropped to $60 \%$ at the next, more salient, step. 
This variability even within a subject may suggest that, at least for some patients with RHD, accurate performance is spurious, or alternatively, that some patients may experience difficulty attending to the task. Again, the RHD subjects experienced more difficulty with the P_BG continua. Whereas the strong performers often achieved $100 \%$ accuracy with the PB_G continua, maximum performance never rose above $80 \%$ for the P_BG continua. Moreover, while two subjects (RHD 2 and 5) achieved this maximum performance for the longer pause durations with keyword durations of $364 \mathrm{~ms}$ (P3), the rest of the 'strong' performers required relatively stronger cues to achieve the same level of accuracy (in particular, the longest keyword duration, $443 \mathrm{~ms}$ (P5)). In contrast to these individuals, four other subjects (RHD 1, 4, 6, and 9) failed to show any marked improvement in performance, regardless of cue salience for both P_BG and PB_G continua. The remaining subject (RHD 7) showed a trend toward improved performance with increasing cue salience, especially with the P_BG continua. Consideration of the diagnostic characteristics of each subject failed to suggest any obvious explanations for the individual variations observed in the data for either patient group.

The present experiment investigated the relationship between two temporal prosodic cues in identifying phrasal boundaries - the duration of the preboundary word (vs non-boundary positions) and duration of the pause at phrase boundaries (vs within phrases). Both of these cues have been shown to be important in disambiguating phrasal groupings (Price et al., 1991; Scott, 1982). These two cues were systematically manipulated to determine the critical durations of each necessary for both non-brain-damaged listeners and LHD and RHD patients to accurately identify phrasal boundaries. Recall that it was expected that LHD listeners would require longer durations, and RHD participants, while possibly impaired, would be able to make use of temporal cues in a manner more similar to that of normal listeners.

The results showed that normal controls used both cues in combination to determine the intended phrasal groupings. For both the P_BG and PB_G continua, reasonably consistent responses $(\geqslant 70 \%)$ were obtained with moderate keyword (approximately $363 \mathrm{~ms}$, or step 3 of both continua) and short pause (minimum $40 \mathrm{~ms}$ ) durations. While shorter word durations (steps 1 and 2) were insufficient to disambiguate the phrasal groupings even with the longest phrase boundary pause, listeners were able to accurately identify phrasal groupings when the longest word duration was paired with the $0 \mathrm{~ms}$ pause duration.

Based on previous research suggesting the preferential processing of temporal parameters in the left hemisphere (e.g., Penhune et al., 1996; Robin et al., 1990; Van Lancker \& Sidtis, 1992), the LHD participants were expected to show deficits in the use of these duration cues in determining phrasal groupings. The LHD group did, in fact, show clear deficits in the use of the temporal cues; compared to the performance of the NC subjects, they were unable to identify the phrase boundaries delineated by cues whose durations were more than adequate for unimpaired listeners. However, because they failed to show improvement as cue salience was increased, it was not possible to draw conclusions regarding the sensitivity of these individuals to temporal prosodic cues, or the threshold of perception for either cue.

While it was not unexpected that the RHD patient group would show deficits in prosodic processing (Baum et al., 1997; Blumstein \& Cooper, 1974; Van Lancker \& Sidtis, 1992), what was surprising was the degree of impairment. Although the RHD subjects generally outperformed the LHD subjects, the group differences were not statistically significant. In other words, rather than displaying temporal processing abilities similar to those of unimpaired individuals, the RHD subjects behaved more like listeners believed to have specific deficits in processing this type of acoustic information. Even with the longest duration stimuli (i.e., longest pause with longest keyword duration, displayed in the last data points in graphs of Figs. 1-4), the RHD subjects failed to show normal temporal processing abilities.

Whereas the normal control participants were able to make use of the two temporal cues to identify the phrase boundaries, neither patient group was consistently able to use either cue in boundary identification judgments. Based on the data at hand, it was therefore not possible to determine the temporal threshold required by the brain-damaged patients for phrase boundary identification. A second experiment was thus designed to determine whether further increases in pause durations, beyond those studied in Experiment 1, would permit the brain-damaged patients in both groups to identify the phrasal groupings. Increases in word durations were not further assessed to avoid creating unnatural-sounding stimuli.

\section{Experiment 2}

\subsection{Methods}

\subsubsection{Subjects}

Most of the same individuals as in Experiment 1 served as participants. Three LHD and two RHD patients who were unable to participate in the second experiment were replaced by comparable alternates. These new subjects also passed all the necessary screening tests for inclusion in the study. The characteristics of these subjects appear in Table 1, along with the participants from Experiment 1. 


\subsubsection{Stimuli}

To create a new set of P_BG stimuli, the longest "pink" keyword was followed by pauses ranging from $160 \mathrm{~ms}$ (the longest pause used in Experiment 1) to $480 \mathrm{~ms}$ in $80 \mathrm{~ms}$ steps. These were then concatenated with the original "and," followed by the shortest "black" keyword (to create the greatest contrast), the original "and" and the original "green." Similarly, to create PB_G stimuli, the shortest "pink" and original "and" were followed by the longest "black" and pauses again ranging from 160 to $480 \mathrm{~ms}$, followed by "and" and "green." There were thus a total of 10 stimuli, five intended as P_BG groupings and five intended as PB_G groupings.

\subsubsection{Procedure}

A test tape was made including 10 repetitions of each of the phrasal stimuli in random order. Testing procedures paralleled those in Experiment 1.

\subsection{Results and discussion}

The percentage of correct responses for NC, LHD, and RHD patients for the P_BG and PB_G pause duration continua are displayed in Figs. 5 and 6, respectively. A Group $\times$ Step ANOVA was performed on the raw data for each continuum. Analysis of the P_BG continuum revealed significant main effects for Step $(F(1,104)=6.733, p<.001)$ and $\operatorname{Group}(F(2,26)=$ $5.025, p<.05)$, but no significant interactions. Post hoc analysis using the Newman-Keuls procedure revealed that across the groups, the two pause steps of shortest duration (120 and $240 \mathrm{~ms}$ ) yielded significantly fewer of the intended (i.e., P_BG) responses than the two longest pause durations ( 400 and $480 \mathrm{~ms}$ ), suggesting a trend towards increased accuracy in identifying phrasal groupings with increased salience of the intra-boundary pause, averaged across groups. Post hoc analyses of the

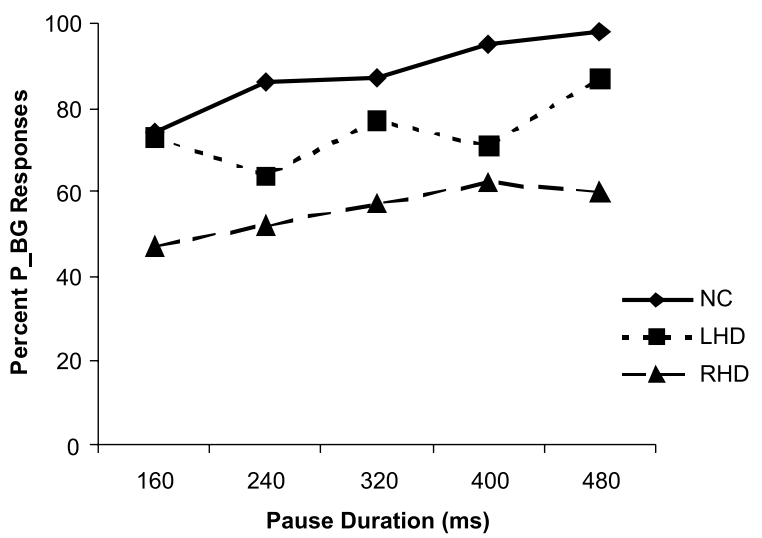

Fig. 5. Percent $\mathrm{P} \_\mathrm{BG}$ responses by NC, RHD, and LHD subjects across the pause duration continuum.

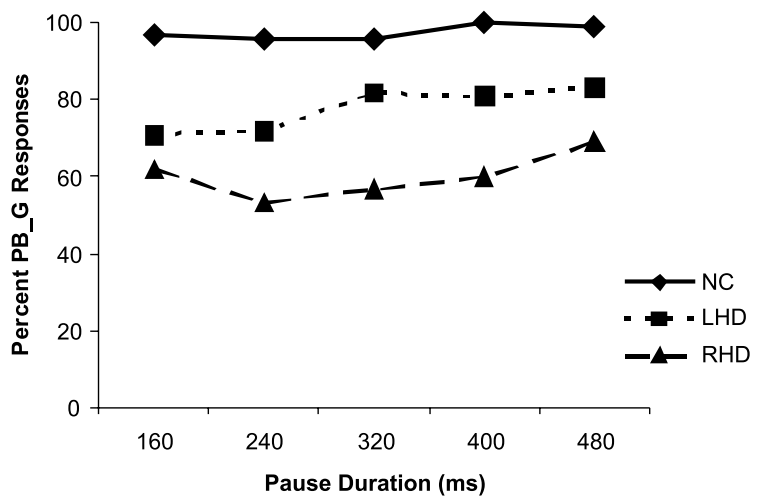

Fig. 6. Percent PB_G responses by NC, RHD, and LHD subjects across the pause duration continuum.

Group main effect showed that normal controls' accuracy was significantly better than that of the RHD patients, but neither differed significantly from the LHD individuals, whose scores fell between these two groups.

Analysis of the PB_G continuum also revealed significant main effects for both factors (Step: $F(4,104)=3.464, p<.05$ and Group: $F(2,26)=$ $169.985, p<.01$ ), but no interaction. Post hoc tests showed that the only significant difference in pause step duration was between the shortest $(160 \mathrm{~ms})$ and longest $(480 \mathrm{~ms})$ steps, suggesting a less-obvious trend towards improved accuracy with increased pause salience. Post hoc analysis of the main effect for Group revealed the same pattern as for the P_BG continuum: normal control subjects outperformed RHD patients, but neither differed from the LHD patient group.

The percentage of "correct" phrase boundary identifications for the LHD group (i.e., responses that were in the direction intended for each continuum) in Experiment 2 ranges from 64 to $87 \%$ for the P_BG series, and from 71 to $83 \%$ for the PB_G series. If these results are compared with the corresponding conditions from Experiment 1, i.e., the longest keyword ("pink" or "black") duration with increasing pause-duration continuum (from 0 to $160 \mathrm{~ms}$ ), where the "correct" responses ranged from 36 to $54 \%$ for the P_BG continuum and from 58 to $72 \%$ for the PB_G continuum, a clear trend towards improved performance with increasing pause durations for the LHD patients becomes apparent. The procedural variations between Experiments 1 and 2 make it difficult to draw firm conclusions regarding this trend. For example, the larger number of repetitions of each stimulus in Experiment 2 (10 repetitions, versus five in Experiment 1) provides increased opportunity for subjects to respond correctly, or perhaps to improve over time, which could account for the findings of improved accuracy in Experiment 2. However, it is more likely that the trend reflects an observable benefit for the LHD patients of the exaggerated 
pause durations in Experiment 2, as neither the RHD nor the NC subjects showed similar trends with the increased number of repetitions.

While the performance of the LHD patients as a group improved with increasing pause durations, the Group $\times$ Step interaction failed to reach significance, as noted above. Individual variability in performance within the LHD group may account for the absence of a significant interaction. Specifically, three of the LHD participants performed at or near ceiling (LHD 2, 7, and 12) from the first pause step $(160 \mathrm{~ms})$ of both continua, while in contrast, two others (LHD 4 and 5) showed little benefit of the increased salience of this cue, and their performance remained low throughout. With these two extremes in performance, then, a trend of improvement with increased cue salience is less obvious in the group data. There are no obvious clinical reasons for these individuals to have performed so differently from the rest of the group. It is conceivable, however, that certain individuals rely more heavily on keyword duration in rendering phrase grouping decisions, whereas others assign more weight to pause duration cues. This hypothesis remains to be verified.

In contrast to the improved abilities of the LHD listeners to identify phrase boundaries given the exaggerated pause, the RHD subjects' accuracy rates did not improve in response to the augmented temporal cue. Their mean identification rates in Experiment 2 ranged from 47 to $69 \%$ "correct" for P_BG and from 53 to $62 \%$ for PB_G, compared to 60 to $71 \%$ (P_BG) and 58 to $67 \%$ (PB_G) in the corresponding conditions in Experiment 1. These results are surprising, given that past research has shown that listeners with right-hemisphere lesions tend to be able to produce and perceive linguistic prosodic cues with better accuracy than LHD individuals (Baum et al., 1982; Behrens, 1988; Emmorey, 1987; Gandour et al., 1993, 1995, 1992; Pell \& Baum, 1997; but cf. Blumstein \& Cooper, 1974; Brådvik et al., 1991), particularly if the contrasts are signaled by temporal parameters (Robin et al., 1990; Tallal \& Newcombe, 1978; Van Lancker \& Sidtis, 1992). However, the RHD patients in the current investigation failed to show the expected pattern in both experiments.

Individual variability in performance profiles was also observed within the RHD patient group. Two of the RHD participants (RHD 2 and 5) performed at or near ceiling on both continua, and two additional subjects performed at ceiling on the PB_G continuum alone (RHD 3 and 11). In contrast, four subjects from this group (RHD 1, 4, 9, and 10) performed very poorly regardless of the increase in pause durations, suggesting that they were not able to use the temporal cue to assist in their boundary decisions. Again, there are no obvious clinical reasons, based on the diagnostic characteristics of these patients, for the performance patterns observed for individual subjects.

\section{General discussion}

The goal of the present study was to assess the sensitivity of individuals with left- or right-hemisphere brain damage, relative to normal controls, in perceiving the temporal cues marking phrase boundaries. The two relevant cues-increase in the duration of the preboundary word and of the pause at the boundary (Lehiste, Olive, \& Streeter, 1976; Price et al., 1991; Scott, 1982; Streeter, 1978) -were independently manipulated, to determine the relative thresholds of each needed for identifying the intended phrase groupings of "pink and black and green" (PBG, P_BG, and PB_G). As expected, non-brain-damaged individuals were sensitive to the temporal manipulations; even when the durations were significantly shorter than those that occur in citation-form speech, unimpaired listeners were able to correctly identify the intended phrasal groupings, especially when both cues were used in combination.

Given the evidence found throughout the literature of impairments in temporal processing following LH brain damage (Robin et al., 1990; Tallal \& Newcombe, 1978; Van Lancker \& Sidtis, 1992), it was expected that the LHD patients would have higher thresholds for perceiving durational prosodic cues. The combined results from the two experiments presented here are consistent with this expectation. In Experiment 1, LHD listeners, as a group, were not able to consistently identify phrasal groupings when presented with stimuli for which nonimpaired individuals performed near ceiling. However, when the pause durations were exaggerated beyond normal levels in Experiment 2, the performance of the LHD patient group improved (although still not to levels comparable to those of the normal controls), suggesting that, in general, LHD patients may exhibit a lowered sensitivity (i.e., higher threshold) to such cues, rather than an overall inability to perceive them, or to map them to linguistically significant contrasts (see, e.g., Johnsrude et al., 2000; Robin et al., 1990).

Recall, however, that individual variability within the LHD group was evident. Following Experiment 2, there were three subjects performing consistently at ceiling, two of whom had continued this high level of performance from Experiment 1 (LHD 2 and 7), and one who was a replacement subject (LHD 12) who only participated in the second study. In addition, there were two subjects (LHD 4 and 5) who showed no improvement at all between Experiments 1 and 2, despite the augmented temporal cues. Nevertheless, half of the LHD patients (LHD 1, 9, 10, 11, and 13) showed clear improvements in their ability to identify phrasal groupings as salience of the temporal cues increased: LHD 1, 9, and 10 showed this improvement between Experiments 1 and 2, while LHD 11 and 13, who were replacement subjects introduced in Experiment 2, gradually increased their accuracy with the progressively longer-duration inter- 
phrase pauses presented within Experiment 2. In other words, most of those LHD patients who had shown deficits in temporal processing exhibited improved performance when the relevant temporal parameters were exaggerated beyond durations shown to be adequate for normal listeners. While the findings here of temporal processing deficits in many of the LHD patients are consistent with most of the data reported in the literature (e.g., Baum et al., 1997; Johnsrude et al., 2000; Robin et al., 1990; Van Lancker \& Sidtis, 1992), the present set of experiments extends these findings by supporting suggestions that the temporal processing deficit following left hemisphere damage reflects a shift in the threshold of perception of temporal parameters rather than an inability to process them or assign them to linguistically significant prosodic contrasts.

As noted earlier, the results for the RHD group were somewhat unexpected. Although some degree of impairment in the identification of phrase boundaries relative to normal was expected given past findings (e.g., Baum et al., 1997), it was nevertheless anticipated that the thresholds at which the pause and keyword durations would yield reasonably consistent phrase boundary identifications would be closer to normal, given the widely held theory that RHD patients are better able to make use of temporal cues to prosody (e.g., Van Lancker \& Sidtis, 1992). In Experiment 1, where shorterthan-normal durations were manipulated, the RHD patients performed equally inaccurately to the LHD patients. Moreover, when pause durations were manipulated to exceed normal values in Experiment 2, the RHD group was still unable to make use of this cue to improve their accuracy. Results from both experiments, then, suggest a deficit on the part of the RHD patients in the ability to identify phrasal groupings based on temporal cues. Examination of the performance of the individual subjects within this patient group reinforces this conclusion. Of all RHD subjects who performed poorly in Experiment 1, only one (RHD 6) showed improvement across experiments as temporal cues were increased. (RHD 7 had shown some improvement within Experiment 1, but because this subject did not participate in Experiment 2, we were unable to conclude with any certainty that performance would have continued to improve with exaggerated cue durations.) This is in contrast to the LHD patients' performance, where most of those who experienced difficulty in processing the temporal prosodic information increased the accuracy of their performance with stronger durational cues. Even among those RHD patients who performed well (RHD 2, 3, and 5 across experiments; RHD 8 in Experiment 1; RHD 11 in Experiment 2), there was marked variability in performance within the individual, suggesting that some RHD patients are unable to perform the task accurately on a consistent basis, or alternatively, that some other mechanism, such as attention deficits, may interfere with otherwise intact processing abilities.

Despite the fact that the overall performance of the RHD group was poorer than expected, it is nevertheless clear that the LHD and RHD populations are distinguishable based on their performance profiles. In Experiment 1, the RHD patients showed a slight tendency to outperform the LHD patients in temporal prosodic processing. In Experiment 2, the LHD group improved with increasing cue duration, while the RHD group failed to show differential responding with increased cue salience. The difference in group response patterns suggests that there are most likely different mechanisms underlying the difficulties experienced by the two patient groups.

As described in Section 1, different theories have been put forth to explain the prosodic deficits observed subsequent to left versus right hemisphere brain damage. While it has generally been established that the neuroanatomical regions subserving prosodic processing are distributed in both cerebral hemispheres, it remains unclear which aspects of prosody are controlled where. The functional lateralization hypothesis (Van Lancker, 1980) asserts that linguistic prosody, such as lexical and emphatic stress, phonemic tone, declarative/interrogative sentence intonation, and syntactic disambiguation (including phrase grouping identification), is mediated by the $\mathrm{LH}$, whereas emotional prosody is mediated by the RH. Many studies have, in fact, shown more deficits in processing linguistic cues subsequent to LHD (Baum et al., 1982; Emmorey, 1987; Gandour et al., 1992; Pell \& Baum, 1997) and fewer deficits following RHD (Behrens, 1988; Emmorey, 1987; Gandour et al., 1993, 1995). A more recent theory, the differential cue lateralization hypothesis (Van Lancker \& Sidtis, 1992) posits that individual acoustic cues are differentially lateralized, with spectral information, such as $F_{0}$, processed by the RH and temporal parameters processed by the LH. There are both behavioural (Carmon \& Nachshon, 1971; Robin et al., 1990; Tallal \& Newcombe, 1978; Van Lancker \& Sidtis, 1992) and neurofunctional (LiégeoisChauvel et al., 1999, 2001; Zatorre, 1988; Zatorre et al., 1992) studies which have supported this view, as well. For example, anatomical differences between the left and right auditory cortices have been found, suggesting the possibility of different processing functions of the two hemispheres (Penhune et al., 1996). Moreover, neuroimaging and physiological studies have recently supported left hemisphere superiority for temporal processing, while spectral processing was more lateralized to the right hemisphere (e.g., Johnsrude et al., 2000; Liégeois-Chauvel et al., 1999, 2001; Zatorre, 1988). It was anticipated that the specific manipulations of temporal prosodic cues within a restricted linguistic function (phrase boundary disambiguation) in the present investigation would have allowed the issue of 
lateralization of temporal prosodic information to be directly addressed.

In particular, the purpose of the current study was to determine whether individuals with LHD show specific deficits in processing temporal prosodic information in linguistic stimuli. If so, the experimental manipulations should have resulted in increased accuracy of performance on the task with increases in temporal cue salience for the LHD subjects, relative to that of normal controls. This was, in fact, the pattern that emerged for the LHD patients; we were furthermore able to characterize the nature of the deficit as a decreased sensitivity, or higher threshold of perceptibility, of the temporal cues to linguistic prosody, as opposed to a general inability to perceive the cues, or to associate them with meaningful linguistic contrasts (Johnsrude et al., 2000; Robin et al., 1990). These results, then, may be interpreted as consistent with the lateralization of processing of (linguistic) temporal prosodic cues to the left hemisphere, or at least with a significant role for the LH in temporal prosodic processing (Carmon \& Nachshon, 1971; Johnsrude et al., 2000; LiégeoisChauvel et al., 1999, 2001; Penhune et al., 1996; Robin et al., 1990; Tallal \& Newcombe, 1978; Van Lancker \& Sidtis, 1992; Zatorre, 1988; Zatorre et al., 1992).

Nonetheless, given that the RHD patients also showed temporal processing deficits, it is difficult to posit complete lateralization of temporal cue. To do so, one would have expected these subjects' performance to mirror that of the normal controls. However, our interpretation of the findings for the RHD subjects does not preclude substantial lateralization of temporal processing. As discussed above, the fact that the RHD group generally failed to improve with increased cue salience suggests that there may be some reason other than temporal processing deficits underlying their poor performance. The results for the RHD group (i.e., difficulty with a linguistic prosodic task) are also not consistent with the functional lateralization hypothesis, although it is difficult to draw conclusions from the present data, as one may only speculate as to the reason for the poor performance of the RHD group. One possible explanation is that these individuals have particular difficulty attending to the task, resulting in reduced accuracy in performance. A second possibility relates to the difficulty that some RHD patients have with selecting from among multiple response alternatives (Leonard, 1994). In the present experiment, listeners were required to choose from among three response alternatives: P_BG, PB_G or PBG. Perhaps had a two-alternative forced-choice paradigm been used, the performance of the RHD patients might have been more in keeping with that of the normal controls. If either of these alternatives-attention deficits or difficulty choosing among multiple response alternativesunderlies the poor performance of this subject group, a functional lateralization for prosodic processing would not be precluded, as the impairment would not be specifically linguistically based.

A final possibility is that the observed deficits in the RHD patients may signify a reliance of these individuals on multiple cues in processing prosody (e.g., Baum, 1998). The stimuli used in the present experiment contained very few prosodic cues, being very short phrases, with little variation in $F_{0}$ or amplitude. In fact, $F_{0}$ has been suggested as the primary cue to prosodic information (e.g., Lieberman, 1960), with temporal alterations providing secondary (albeit important) information (Klatt, 1976; Lyberg, 1979). If RHD patients require a combination of prosodic cues to accurately process stimuli, the lack of such important information in the present stimuli (coupled with their supposed deficits in processing what little spectral information there may be; Van Lancker \& Sidtis, 1992) may be masking these patients' otherwise intact ability to process temporal cues. Future research focusing on the need for multiple prosodic cues in RHD populations would provide the necessary information to resolve this issue.

An unpredicted, yet interesting, additional finding was obtained with the two different phrasal groupings of the stimulus words. Specifically, subjects in all experimental groups (normal controls, LHD, and RHD) were generally better at disambiguating the PB_G continua relative to the $\mathrm{P}_{-} \mathrm{BG}$ continua; the same pattern was found in both experiments. The reason for this bias favouring the PB_G grouping is not clear. Perhaps it reflects a contextual rate normalization effect. If temporal cues as to the syntactic structure of an utterance, such as those indicating phrase boundaries, are interpreted relative to the overall temporal characteristics or rate of the utterance, then any given cue would be easier to interpret when a rate context has already been established. In the case of the phrase boundaries studied here, the cues (pre-boundary word durations and inter-phrase pauses) may be more easily interpretable when preceded by a phrase providing multiple cues to the overall rate of the utterance. In other words, perhaps there are more cues as to the relative temporal envelope of the utterance in the PB_G versus the P_BG conditions. For example, when the PB_G grouping is heard, there is no pause between "pink-and-black," the word "black" is lengthened (and "pink" is not), and an obvious pause is heard following this string of words before the utterance is completed ("... and green"). When P_BG is heard, however, while the two dominant cues as to phrase grouping are present (i.e., the word "pink" is lengthened and followed by a pause), the listener does not yet know whether this necessarily indicates phrasal separation from what is yet to be uttered, or simply a slow overall speaking rate. Whichever possibility is correct is not known until the utterance is 
complete (or at least until adequate cues to speaking rate have been presented) when the listener may need to reanalyze, or "normalize" the utterance, to determine the relative temporal cues indicating phrasal grouping. Thus the PB_G continua may be easier to disambiguate because there are more temporal cues available earlier in the utterance, making the phrasal grouping more obvious initially, and obviating the need to potentially reanalyze the sentence. A second alternative is that the apparent bias may be due to a differential effect of the keyword manipulations. Specifically, because the [I] in "pink" is inherently quite short, there are limits to the extent to which it can be lengthened or extended and retain its natural sound quality. In contrast, whereas the [æ] in "black" is also inherently a lax vowel, it tends to be much longer in duration and to pattern with long vowels in rate manipulations (Baum, 1993). As a result, the P_BG continua may have sounded somewhat more unnatural in comparison to the PB_G continua, leading to slightly poorer identification performance across the board.

In conclusion, the data from the present experiments show that, whereas normal listeners are able to use temporal cues indicating phrase boundaries (i.e., increased durations of the pre-boundary word and of the following inter-phrase pause) to identify phrasal groupings, both LHD and, surprisingly, RHD patients (as a group) had significant difficulty processing this temporal information. Increasing the length of the pause beyond normal values allowed the LHD patient group to accurately perform the task, suggesting that their deficits are mediated by a shift to a higher perceptual threshold for temporal prosodic information in spoken language. Increasing the salience of the temporal cues had no effect on the RHD patient group's performance, suggesting that their difficulties may not be related to temporal processing, but rather to some other mechanism, such as the limited acoustic cues available in the stimuli. Limited support for the lateralization of processing of linguistic temporal prosody to the left hemisphere, in keeping with the differential cue lateralization hypothesis (Van Lancker \& Sidtis, 1992), emerged in the current investigations. Further specification as to the nature of right hemisphere deficits in this and similar tasks (e.g., Baum et al., 1997) is necessary to be able to draw more firm conclusions regarding the neural substrates for linguistic temporal prosody.

\section{Appendix A}

Illustration of continua derived from keyword- and pause-duration manipulations, using the P_BG grouping as an example. Note that 10 different continua are created, each with five steps: five "pause duration" continua, in which the pause steps vary across each level of keyword step; and five "keyword duration" continua, in which the keyword steps vary across each level of pause step.

\begin{tabular}{|c|c|c|}
\hline $\begin{array}{l}\text { Keyword } \\
\text { "Pink" steps }\end{array}$ & $\begin{array}{l}\text { Pause steps } \\
\text { (ms) }\end{array}$ & \\
\hline $\mathrm{P} 1$ & $\leqslant$ & $\begin{array}{l}+\quad \text { "and black } \\
\text { and green." }\end{array}$ \\
\hline $\mathrm{P} 2$ & $\aleph_{100}^{i} \Rightarrow$ & $\begin{array}{l}+\quad \text { "and black } \\
\text { and green." }\end{array}$ \\
\hline P3 & $\aleph_{160}^{i} \supsetneq$ & $\begin{array}{l}+\quad \text { "and black } \\
\text { and green." }\end{array}$ \\
\hline P4 & $\aleph_{160}^{i} \nRightarrow$ & $\begin{array}{l}+\quad \text { "and black } \\
\text { and green." }\end{array}$ \\
\hline P5 & $\aleph_{160}^{i} \Rightarrow$ & $\begin{array}{l}+\quad \text { "and black } \\
\text { and green." }\end{array}$ \\
\hline
\end{tabular}

\section{Appendix B}

Main effects following the Subject Group (NC, LHD, RHD) $\times$ Continuum Step (0, 40, 80, 120, and $160 \mathrm{~ms})$ ANOVAs.

\begin{tabular}{|c|c|c|c|c|c|}
\hline \multicolumn{3}{|c|}{ Keyword "Pink" } & \multicolumn{3}{|c|}{ Keyword "Black" } \\
\hline \multicolumn{6}{|c|}{ Keyword duration continua } \\
\hline \multirow[t]{2}{*}{$\mathrm{P} 1$} & Group & $F(2,26)=4.89, p<.020$ & B1 & Group & $F(2,26)=0.31, p=.70^{*}$ \\
\hline & Step & $F(4,104)=8.16, p<.001$ & & Step & $F(4,104)=11.57, p<.001$ \\
\hline \multirow[t]{2}{*}{$\mathrm{P} 2$} & Group & $F(2,26)=3.14, p=.060^{*}$ & $\mathrm{~B} 2$ & Group & $F(2,26)=0.54, p=.59^{*}$ \\
\hline & Step & $F(4,104)=12.98, p<.001$ & & Step & $F(4,104)=13.20, p<.01$ \\
\hline \multirow[t]{2}{*}{ P3 } & Group & $F(2,26)=10.06, p=.001$ & B3 & Group & $F(2,26)=5.65, p<.01$ \\
\hline & Step & $F(4,104)=10.86, p=<.001$ & & Step & $F(4,104)=4.19, p<.01$ \\
\hline \multirow[t]{2}{*}{ P4 } & Group & $F(2,26)=4.96, p<.02$ & B4 & Group & $F(2,26)=4.17, p<.05$ \\
\hline & Step & $F(4,104)=3.11, p<.02$ & & Step & $F(4,104)=1.29, p .277^{*}$ \\
\hline P5 & $\begin{array}{l}\text { Group } \\
\text { Step }\end{array}$ & $\begin{array}{l}F(2,26)=5.45, p<.02 \\
F(4.104)=4.17 . \quad p<.005\end{array}$ & B5 & $\begin{array}{l}\text { Group } \\
\text { Step }\end{array}$ & $\begin{array}{l}F(2,26)=5.47, p=.01 \\
F(4.104)=1.08, p=.371^{*}\end{array}$ \\
\hline
\end{tabular}


Appendix B. (continued)

\begin{tabular}{llllll}
\hline \multicolumn{2}{l}{ Keyword "Pink" } & \multicolumn{3}{l}{ Keyword "Black" } \\
\hline \multicolumn{2}{l}{ Pause duration continua } & & & \\
P0 & Group & $F(2,26)=4.81, p<.02$ & B0 & Group & $F(2,26)=0.17, p=.85^{*}$ \\
& Step & $F(4,104)=28.16, p<.001$ & & Step & $F(4,104)=38.87, p<.001$ \\
P40 & Group & $F(2,26)=8.51, p=.001$ & B40 & Group & $F(2,26)=4.35, p<.05$ \\
& Step & $F(4,104)=24.22, p<.001$ & & Step & $F(4,104)=34.00, p<.001$ \\
P80 & Group & $F(2,26)=5.52, p=.01$ & \multirow{2}{*}{ B80 } & Group & $F(2,26)=5.43, p<.05$ \\
& Step & $F(4,104)=20.91, p<.001$ & & Step & $F(4,104)=25.65, p<.001$ \\
P120 & Group & $F(2,26)=5.77, p<.01$ & B120 & Group & $F(2,26)=4.18, p<.05$ \\
& Step & $F(4,104)=17.32, p<.001$ & & Step & $F(4,104)=21.62, p<.001$ \\
P160 & Group & $F(2,26)=4.69, p<.02$ & B160 & Group & $F(2,26)=2.24, p=.127^{*}$ \\
& Step & $F(4,104)=14.98, p<.001$ & & Step & $F(4,104)=14.57, p<.001$ \\
\hline
\end{tabular}

${ }^{*}$ Effects which failed to reach significance.

\section{References}

Baum, S. (1993). An acoustic analysis of rate of speech effects on vowel production in aphasia. Brain and Language, 44, 414-430.

Baum, S. (1998). The role of fundamental frequency and duration in the perception of linguistic stress by individuals with brain damage. Journal of Speech, Language, and Hearing Research, 41, $31-40$.

Baum, S., Kelsch Daniloff, J., Daniloff, R., \& Lewis, J. (1982). Sentence comprehension by Broca's aphasics: Effects of some suprasegmental variables. Brain and Language, 17, 261-271.

Baum, S., \& Pell, M. (1999). The neural bases of prosody: Insights from lesion studies and neuroimaging. Aphasiology, 13(8).

Baum, S., Pell, M., Leonard, C., \& Gordon, J. (1997). The ability of right- and left-hemisphere-damaged individuals to produce and interpret prosodic cues marking phrasal boundaries. Language and Speech, 40, 313-330.

Behrens, S. (1988). The role of the right hemisphere in the production of linguistic stress. Brain and Language, 33, 104-127.

Behrens, S. (1989). Characterizing sentence intonation in a right hemisphere-damaged population. Brain and Language, 37, 181200.

Blumstein, S., \& Cooper, W. (1974). Hemispheric processing of intonation contours. Cortex, 10, 146-158.

Brådvik, B., Dravins, C., Holtås, S., Rosén, I., Ryding, E., \& Ingvar, D. (1991). Disturbances of speech prosody following right hemisphere infarcts. Acta Neurologica Scandinavia, 84(2), 114-126.

Bryan, K. (1989). Language prosody and the right hemisphere. Aphasiology, 3(4), 285-299.

Carmon, A., \& Nachshon (1971). Effect of unilateral brain damage on perception of temporal order. Cortex, 7, 410-418.

Efron, R. (1963). Temporal perception, aphasia and déjàvu. Brain, 86, 403-424.

Emmorey, K. (1987). The neurological substrates for prosodic aspects of speech. Brain and Language, 30(2), 305-320.

Gandour, J., Dechongkit, S., Ponglorpisit, S., Khunadorn, F., \& Boongird, P. (1993). Intraword timing relations in Thai after unilateral brain damage. Brain and Language, 45, 160-179.

Gandour, J., Larsen, J., Dechongkit, S., Ponglorpisit, S., \& Khunadorn, F. (1995). Speech prosody in affective contexts in Thai patients with right hemisphere lesions. Brain and Language, 51, 422-443.

Gandour, J., Petty, S., \& Dardarananda, R. (1989). Dysprosody in Broca's aphasia: A case study. Brain and Language, 37, 232-257.

Gandour, J., Ponglorpisit, S., Khunadorn, F., Dechongkit, S., Boongrid, P., \& Boonklam, R. (1992). Lexical tones in Thai after unilateral brain damage. Brain and Language, 43, 275-307.
Grosjean, F., \& Hirt, C. (1996). Using prosody to predict the end of sentences in English and French: Normal and brain-damaged subjects. Language and Cognitive Processes, 11, 107-134.

Johnsrude, I. J., Penhune, V. B., \& Zatorre, R. J. (2000). Functional specificity in the right human auditory cortex for perceiving pitch direction. Brain, 123, 155-163.

Klatt, D. (1976). Linguistic uses of segmental duration in English: Acoustic and perceptual evidence. Journal of the Acoustical Society of America, 59, 1208-1221.

Langner, G., Sams, M., Heil, P., \& Schulze, H. (1997). Frequency and periodicity are represented in orthogonal maps in the human auditory cortex: evidence from magnetoencephalography. Journal of Comparative Physiology A: Sensory Neural and Behavioral Physiology, 181(6), 665-676.

Lehiste, I., Olive, J., \& Streeter, L. (1976). Role of duration in disambiguating syntactically ambiguous sentences. Journal of the Acoustical Society of America, 60, 1199-1202.

Lieberman, P. (1960). Some acoustic correlates of word stress in American English. Journal of the Acoustical Society of America, 32, 451-454.

Liégeois-Chauvel, C., de Graaf, J., \& Laguitton, V., et al. (1999). Specialization of left auditory cortex for speech perception in man depends on temporal coding. Cerebral Cortex, 9, 484-496.

Liégeois-Chauvel, C., Giraud, K., Badier, J. M., Marquis, P., \& Chauvel, P. (2001). Intracerebral evoked potentials in pitch perception reveal a functional asymmetry of the human auditory cortex. Annals of the New York Academy of Sciences, 930, 117-132.

Leonard, C. (1994). The use of contextual information by right brain damaged individuals in the resolution of ambiguous pronouns. Unpublished doctoral dissertation, McGill University, Montréal, Québec, Canada.

Lyberg, B. (1979). Final lengthening-partly a consequence of restrictions on the speed of fundamental frequency change? Journal of Phonetics, 7, 187-196.

Mertus, J. (1989). BLISS user's manual. Providence: Brown University.

Pell, M., \& Baum, S. (1997). The ability to perceive and comprehend intonation in linguistic and affective contexts by brain damaged adults. Brain and Language, 57, 80-99.

Penhune, V. B., Zatorre, R. J., \& MacDonald, J. D., et al. (1996). Interhemispheric anatomical differences in human primary auditory cortex: Probabilistic mapping and volume measurement from magnetic resonance scans. Cerebral Cortex, 6, 661-672.

Perkins, J., Baran, J., \& Gandour, J. (1996). Hemispheric specialization in processing intonation contours. Aphasiology, 10, 343-362.

Price, P., Ostendorf, M., Shattuck-Hufnagel, S., \& Fong, C. (1991). The use of prosody in syntactic disambiguation. Journal of the Acoustical Society of America, 90, 2956-2970. 
Robin, D. A., Tranel, D., \& Damasio, H. (1990). Auditory perception of temporal and spectral events in patients with focal left and right cerebral lesions. Brain and Language, 39, 539-555.

Scott, D. (1982). Duration as a cue to the perception of a phrase boundary. Journal of the Acoustical Society of America, 71, 996-1007.

Streeter, L. (1978). Acoustic determinants of phrase boundary perception. Journal of the Acoustical Society of America, 64, 1582-1592.

Tallal, P., \& Newcombe, F. (1978). Impairment of auditory perception and language comprehension in dysphasia. Brain and Language, 5, $13-24$.

Van Lancker, D. (1980). Cerebral lateralization of pitch cues in the linguistic signal. International Journal of Human Communication, 13(2), 227-277.
Van Lancker, D., \& Sidtis, J. (1992). The identification of affectiveprosodic stimuli by left- and right-hemisphere-damaged subjects: All errors are not created equal. Journal of Speech and Hearing Research, 35, 963-970.

Weintraub, S., Mesulam, M.-M., \& Kramer, L. (1981). Disturbances in prosody: A right-hemisphere contribution to language. Archives of Neurology, 38, 742-744.

Zatorre, R. (1988). Pitch perception of complex tones and human temporal-lobe function. Journal of the Acoustical Society of America, 84, 566572.

Zatorre, R., Evans, A., Meyer, E., \& Gjedde, A. (1992). Lateralization of phonetic and pitch discrimination in speech processing. Science, 256, 846-849. 